\title{
Impact of hypertension on short- and long-term survival of patients who underwent emergency surgery for type $A$ acute aortic dissection
}

\author{
Zhigang Wang, Min Ge, Tao Chen, Cheng Chen, Qiuyan Zong, Lichong Lu, Dongjin Wang \\ Department of Cardio-thoracic Surgery, Nanjing Drum Tower Hospital, The Affiliated Hospital of Nanjing University Medical School, Nanjing, \\ China \\ Contributions: (I) Conception and design: D Wang; (II) Administrative support: D Wang; (III) Provision of study materials or patients: T Chen, C \\ Chen, Q Zong; (IV) Collection and assembly of data: Z Wang, L Lu; (V) Data analysis and interpretation: Z Wang, T Chen, M Ge; (VI) Manuscript \\ writing: All authors; (VII) Final approval of manuscript: All authors. \\ Correspondence to: Dongjin Wang. Department of Cardio-thoracic Surgery, Nanjing Drum Tower Hospital, The Affiliated Hospital of Nanjing \\ University Medical School, Nanjing 210008, China. Email: glyywdj@163.com.
}

Background: To explore the clinical characteristics and prognosis of patients with hypertension underwent emergency surgery for type A acute aortic dissection (TA-AAD).

Methods: The present study enrolled 712 consecutive patients diagnosed with TA-AAD and received aortic repair surgery at our hospital between January 2014 to December 2018. Clinical characteristics of enrolled patients were retrospectively reviewed. Patients were grouped by preexisting hypertension history and matched with propensity scores matching method. Patients' clinical characteristics were compared and analyzed before and after propensity scoring. To identify predictors for long-term mortality rate, KaplanMeier survival estimation and Cox proportional hazard analysis were performed.

Results: A total of 492 patients $(69.1 \%$ of all patients in the cohort) were included in the hypertensive group and they had increased age and weight compared to patients in the non-hypertensive group. Between two groups, preoperative leukocyte count and serum creatinine level were found significant different $(\mathrm{P}<0.05)$. After propensity scoring, 128 pairs (256 patients) were successfully matched. Our analysis showed that there was no significant difference of ventilation duration, 30-day mortality rate, intensive care unit stay and hospitalization time between two groups. However, our data suggested that hypertensive patients presented with less intra-operative aortic valve involvement. There was a significant difference in long-term survival rate $(\mathrm{P}=0.037)$ between two groups. Cox regression analysis demonstrated that hypertension was an independent risk factor [hazard ratio (HR), 3.040; 95\% confidence interval (CI), 1.124-8.227; P=0.029].

Conclusions: Our data suggested that TA-AAD patients complicated with hypertension had increased age and weight compared to non-hypertensive patients. Concomitant hypertension identified upon hospital administration was an independent risk factor for long-term survival in TA-AAD patients while did not influence the 30-day mortality rate.

Keywords: Type A acute aortic dissection; hypertension; early outcomes; propensity score matching; long-term outcomes

Submitted Jun 30, 2020. Accepted for publication Sep 30, 2020.

doi: $10.21037 /$ jtd-20-2336

View this article at: http://dx.doi.org/10.21037/jtd-20-2336 


\section{Introduction}

Type A acute aortic dissection (TA-AAD) is a lifethreatening disease, characterized by abrupt onset and catastrophic outcomes. The mortality rate of acute aortic dissection increases by $1 \%$ to $2 \%$ per hour after the first 24 to $48 \mathrm{~h}$ of symptom onset (1) and ranges from $8 \%$ to $34 \%$ even after prompt surgeries (2). Several risk factors associated with spontaneous dissection have been identified and hypertension is considered the most important one and presented in about $65-75 \%$ of all patients (3). In general population, hypertension accounts for $54 \%$ of the population-attributable risk of developing acute aortic dissection (4).

Surgical repair is the $1^{\text {st }}$ line treatment for TAAAD patients. Repair of TA-AAD can be restrained to replacement of the proximal arch sparing the aortic arch branch vessels or total arch replacement. Study showed that the prevalence of hypertension in thoracic aortic dissection patients increased in the past few years (5). However, even though the importance of hypertension in preoperative risk evaluation has been well examined, whether hypertension could influence short- and long-term survival rates after surgery has not been well studied. Therefore, the present study aimed to investigate the impact of pre-existing hypertension on short- and long-term mortality rates in patients with TA-AAD after emergency surgery. We present the following article in accordance with the STROBE reporting checklist (available at http://dx.doi.org/10.21037/ jtd-20-2336).

\section{Methods}

\section{Patients}

A total of 712 consecutive patients diagnosed with TA-AAD and received aortic repair surgery at our hospital between January 2014 to December 2018 were initially screened for the study. The diagnosis of aortic dissection was made using computed tomography angiography upon admission to the hospital. This study was reviewed and approved by the institutional research ethics committee of Nanjing Drum Tower Hospital (No. BL2014004). Informed written consent was waived owing to the retrospective nature of the study. The study was conducted in accordance with the Declaration of Helsinki (as revised in 2013).

Exclusion criteria included a history of preoperative renal replacement therapy or death occurred within 24 hours after surgery. All patients were assigned to two groups based on if they were also diagnosed with hypertension upon hospital admission.

\section{Date collection}

A standardized form was used to collect clinical information. Data included patient demographics, history of hypertension and aortic dissection disease, blood pressure (BP) at admission, aortic dissection features (blood supply of renal artery), peri-operational parameters including preoperative pericardial effusion, operation parameters and postoperative parameters like drainage volume 24 hours after surgery, mechanical ventilation time, 30-day mortality, length of intensive care unit (ICU) stay, and hospitalization time were collected.

\section{Outcome variables}

The primary endpoint was long-term survival rates with a median follow-up of 29 months. Secondary endpoints were early postoperative clinical characteristics and surgery complications.

\section{Surgical techniques}

A standard median sternotomy was routinely performed on patients with TA-AAD. After systemic heparinization, cardiopulmonary bypass (CPB) was established by arterial cannulation of the right axillary or femoral artery or the ascending aorta and venous cannulation of the right atrium. When patients were cooled to $18-20{ }^{\circ} \mathrm{C}$ (nasopharyngeal temperature) or $22-24{ }^{\circ} \mathrm{C}$ (bladder temperature), the blood circulation was stopped, and the flow rate was reduced to $3-5 \mathrm{~mL} / \mathrm{kg} / \mathrm{min}$. After clamping of the ascending aorta, cardiac arrest was accomplished with cold cardioplegic solution. Bentall procedure or ascending aorta replacement was performed depending on the conditions of aortic regurgitation. Total arch replacement method was traditionally selected in the following scenarios: major dissection tear around the aortic arch or proximal descending aorta, or diameter of the aortic arch is greater than $4 \mathrm{~cm}$ on contrast computed tomography. Once the distal and transverse anastomoses were completed, antegrade perfusion was reinitiated. Systemic warming was then initiated, and the proximal reconstruction marked the completion of a standard $\mathrm{CPB}$ surgery. All patients were then transferred to ICU for routine monitoring. 


\section{Follow-up}

Routine evaluation of enrolled patients' general health status was obtained through contacting with patients and their relatives through phone calls which was conducted every one year after discharging from the hospital. The follow-up information included BP, management of hypertension, current medication and deaths (if any, cause of death and date were recorded). All follow-up data was collected. The median follow-up period was 29 months, and follow-up information was obtained in $92.1 \%$ (569 of 618) of all enrolled patients.

\section{Diagnosis of hypertension}

For TA-AAD patients, the diagnosis of hypertension was made if the patient was diagnosed with hypertension by a physician previously or presented with at least 3 times of elevated BP (systolic BP greater than $140 \mathrm{mmHg}$ and diastolic BP greater than $90 \mathrm{mmHg}$ ) on different days during hospitalization (6).

\section{Statistical analysis}

Data was analyzed with SPSS software (version 25.0, IBM Corp, Armonk, NY). Continuous and categorical variables were expressed as mean \pm standard deviation. The student $t$-test was used for Gaussian distributed continuous variables and the Mann-Whitney $U$ test for non-Gaussian distributed variables. Categorical variables were compared with the $\chi^{2}$ test or Fisher exact test.

To reduce selection bias, a one-to-one propensity score matching (PSM) method was applied to analyze the short-term outcomes, with hypertension as the dependent variable. And it was conducted between groups highlighted in the univariate analysis (preoperative characteristics that showed significant differences between two groups). PSM was based on one-to-one nearest neighbor matching method with a tolerance level on the maximum propensity score distance (calipers of width 0.1 standard deviations of the logit of the propensity score).

Kaplan-Meier survival estimation was applied for longterm survival analysis, whereas the Log-Rank test was applied for the calculation of significances for followup period. Univariate and multivariate Cox proportional hazard analysis were performed to determine independent predictors for late mortality, variables with a $\mathrm{P}$ value less than 0.1 on univariate analyses were included in the multivariate model. All analyses were two-sided, and any $\mathrm{P}<0.05$ was considered statistically significant.

\section{Results}

\section{Demographic and clinical features}

After excluding 3 patients requiring dialysis and 15 patients with death within 24 hours after the operation, a total of 712 patients were included in this study. Among these patients, 694 patients $(97.5 \%)$ received emergency operation and 492 patients (69.1\%) were diagnosed with hypertension. Patients' preoperative characteristics were presented in Table 1. Compared to normotensive patients, patients with hypertension were elder and heavier $(\mathrm{P}<0.001)$ and presented with elevated preoperative leucocyte and serum creatinine $(\mathrm{sCr})$ levels.

In terms of operative variables, hypertensive patients required less intraoperative aortic valve management and experienced shorter aortic cross-clamp duration. While the posterior parallel circulation time and the deep hypothermic circulatory arrest duration were significantly longer in the hypertensive group, as shown in Table 2. In addition, normotensive patients were more likely to receive aortic valve management $(\mathrm{P}<0.001)$.

In order to better compare patients with similar baseline characteristics, propensity matching was performed in the entire cohort based on age, body mass index (BMI), preoperative leucocyte count and preoperative sCr. After matching, a total of 256 patients (128 pairs) remained for further analysis.

\section{Short-term outcome}

As indicated in Table 3, hypertensive patients had a significantly higher incidence of developing in-hospital complications compared to patients without hypertension. The 30-day mortality rate before propensity matching was $14.4 \%$ in the hypertension group and $8.6 \%$ in the normotensive patients $(\mathrm{P}=0.032)$. Other parameters, such as average ICU stay and hospital stay showed no significant differences between two groups.

However, there was no significant differences discovered between hypertensive and normotensive patients regarding 30 -day mortality $(9.4 \%$ vs. $10.9 \%, \mathrm{P}=0.697)$ after propensity-matching suggested that the increased mortality observed in hypertensive group previously was largely due to the patients' baseline differences. Furthermore, our data suggested that there was no difference in variables like 
Table 1 Comparison of preoperative variables before and after matching

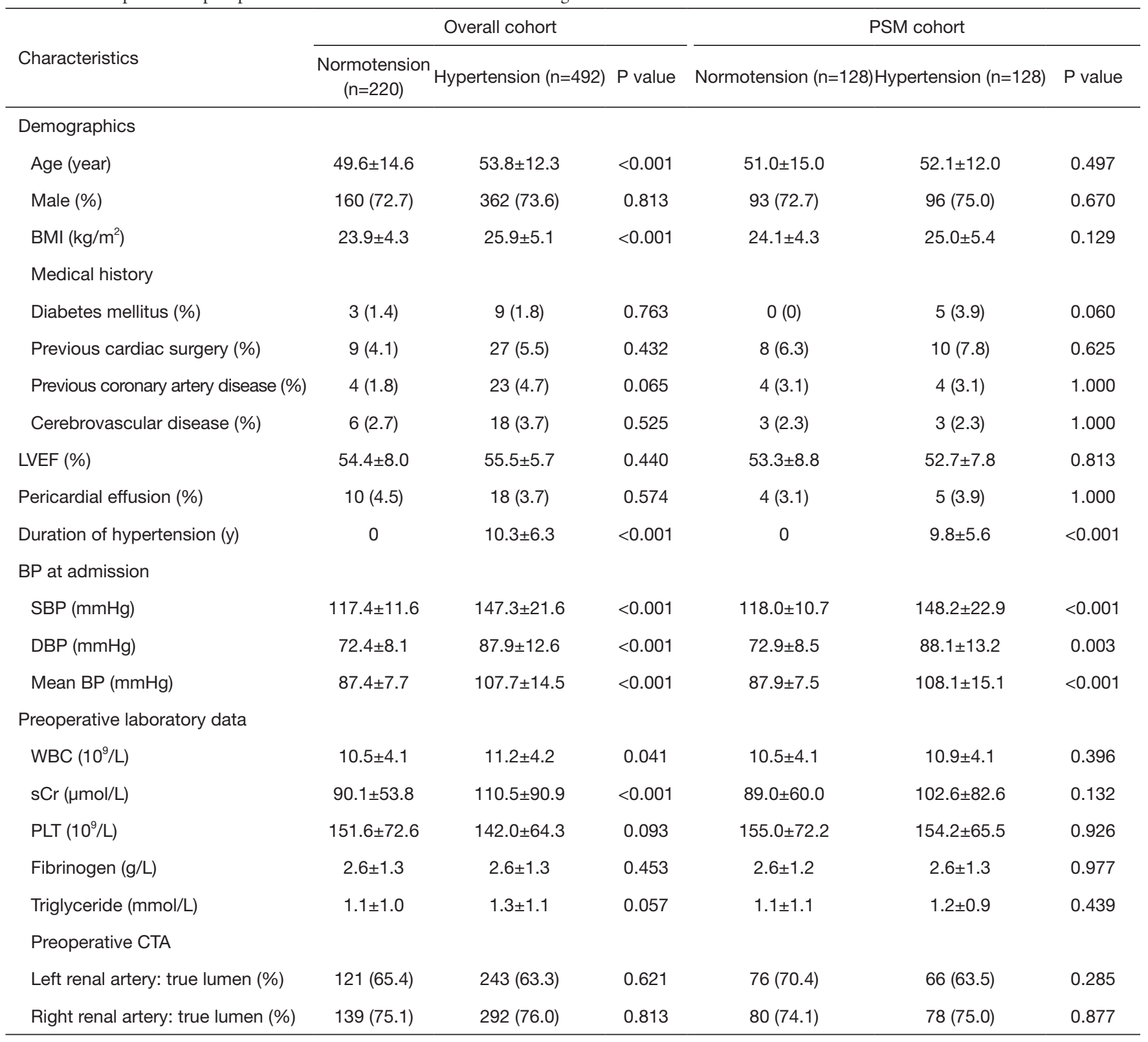

Data presented as $\mathrm{n}(\%)$; mean \pm standard deviation. PSM, propensity score matching; BMI, body mass index; LVEF, left ventricular ejection fraction; ACEI, angiotensin-converting enzyme inhibitors; ARB, angiotensin receptor blocker; BP, blood pressure; SBP, systolic blood pressure; DBP, diastolic blood pressure; WBC, white blood cell; SCr, serum creatinine; PLT, platelet; CTA, computed tomography angiography.

ventilation duration, ICU and hospital stay time between two matched groups.

\section{Long-term outcome}

Seventy-three patients (14.8\%) in the hypertensive group and 21 patients $(9.5 \%)$ in the normotensive group died during the hospitalization period. As a result, a total of 419 hypertensive patients and 199 normotensive patients were included in the follow-up. We identified $32(6.5 \%)$ deaths that occurred in the hypertension group and $9(4.1 \%)$ in the normotensive group. The causes of late death are presented 
Table 2 Comparison of operative variables before and after matching

\begin{tabular}{|c|c|c|c|c|c|c|}
\hline Characteristics & \multicolumn{3}{|c|}{ Overall cohort } & \multicolumn{3}{|c|}{ PSM cohort } \\
\hline TAR (\%) & $117(53.2)$ & $254(51.6)$ & 0.701 & $63(49.2)$ & $72(56.3)$ & 0.260 \\
\hline CABG/MVR/MVP/TVP (\%) & $26(11.8)$ & $42(8.5)$ & 0.169 & 17 (13.3) & $13(10.2)$ & 0.437 \\
\hline $\begin{array}{l}\text { Posterior parallel circulation time } \\
\text { (min) }\end{array}$ & $51.4 \pm 28.4$ & $58.1 \pm 34.4$ & 0.008 & $48.5 \pm 21.1$ & $54.2 \pm 25.1$ & 0.055 \\
\hline Aortic cross-clamp time (min) & $176.0 \pm 56.7$ & $165.1 \pm 58.6$ & 0.021 & $171.4 \pm 55.7$ & $171.2 \pm 66.6$ & 0.976 \\
\hline
\end{tabular}

Data presented as $\mathrm{n}(\%)$; mean \pm standard deviation. PSM, propensity score matching; TAR, total arch replacement; CABG, coronary artery bypass graft; MVR, mitral valve replacement; MVP, mitral valvuloplasty; TVP, tricuspid valvuloplasty; CPB, cardiopulmonary bypass; DHCA, hypothermic circulatory arrest.

Table 3 Comparison of postoperative variables before and after matching.

\begin{tabular}{|c|c|c|c|c|c|c|}
\hline Characteristics & \multicolumn{3}{|c|}{ Overall cohort } & \multicolumn{3}{|c|}{ PSM cohort } \\
\hline $\begin{array}{l}\text { Drainage volume } 24 \\
\text { hours after surgery }(\mathrm{mL})\end{array}$ & $808.6 \pm 833.9$ & $721.8 \pm 607.0$ & 0.182 & $828.1 \pm 935.0$ & $696.9 \pm 547.2$ & 0.184 \\
\hline Ventilation time (hour) & $33.5 \pm 49.3$ & $35.5 \pm 53.5$ & 0.704 & $36.4 \pm 56.7$ & $33.1 \pm 45.0$ & 0.656 \\
\hline Hospital stay time (day) & $21.6 \pm 11.1$ & $22.7 \pm 13.2$ & 0.301 & $21.3 \pm 13.2$ & $23.9 \pm 13.7$ & 0.128 \\
\hline
\end{tabular}

Data presented as $\mathrm{n}(\%)$; mean \pm standard deviation. PSM, propensity score matching; ICU, intensive care unit.

Table 4 Causes of late mortality $(n=41)$

\begin{tabular}{lcc}
\hline \multirow{2}{*}{ Cause of late mortality } & \multicolumn{2}{c}{ Overall cohort } \\
\cline { 2 - 3 } & Normotension $(\mathrm{n}=9)$ & Hypertension $(\mathrm{n}=32)$ \\
\hline Aortic rupture & 2 & 7 \\
Cardiac failure & 2 & 4 \\
Neurological & 1 & 3 \\
Respiratory failure & & 1 \\
Malignancy & 1 & 1 \\
Suicide & 1 & 16 \\
Unknown & 2 & \\
\hline
\end{tabular}

in Table 4. It was important to point out that 1 normotensive patient committed suicide six months after discharge and was excluded from the study. 48 (7.8\%) patients were lost to follow-up after hospital discharge, and these patients were excluded from the subsequent long-term survival analysis. The preoperative, operative and postoperative characteristics of the 49 patients mentioned above were comparable with the rest of the population (569 patients) (Table 5). Comparison between patients who survived (529 patients) with who did not (40 patients) suggested that the incidence of hypertension in patients who deceased during follow-up was higher and the survived patients were more likely to take regular anti-hypertensive medication (Table 6). 
Table 5 Comparison of variables between lost to follow-up and the rest of patients

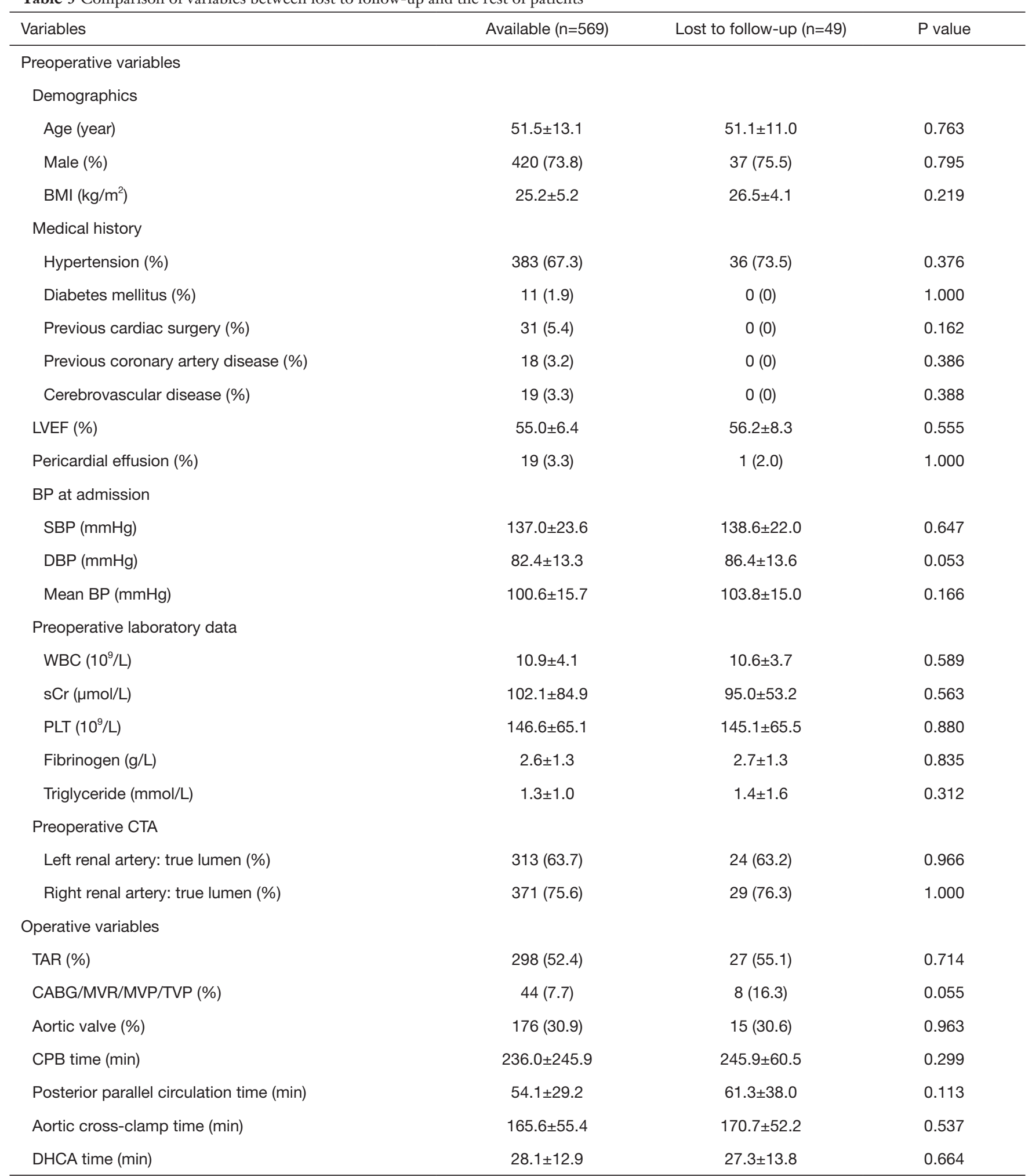

Table 5 (continued) 
Table 5 (continued)

\begin{tabular}{|c|c|c|c|}
\hline Variables & Available $(n=569)$ & Lost to follow-up $(n=49)$ & $P$ value \\
\hline \multicolumn{4}{|l|}{ Postoperative variables } \\
\hline Drainage volume 24 hours after surgery $(\mathrm{mL})$ & $700.7 \pm 601.0$ & $725.0 \pm 629.3$ & 0.792 \\
\hline Ventilation time (hour) & $34.3 \pm 49.2$ & $27.2 \pm 24.6$ & 0.421 \\
\hline ICU stay time (day) & $6.9 \pm 11.9$ & $6.1 \pm 4.9$ & 0.633 \\
\hline
\end{tabular}

Data presented as $\mathrm{n}(\%)$; mean \pm standard deviation. BMI, body mass index; LVEF, left ventricular ejection fraction; LVEF, left ventricular ejection fraction; BP, blood pressure; SBP, systolic blood pressure; DBP, diastolic blood pressure; WBC, white blood cell; PLT, platelet; SCr, serum creatinine; PLT, platelet; CTA, computed tomography angiography; TAR, total arch replacement; CABG, coronary artery bypass graft; MVR, mitral valve replacement; MVP, mitral valvuloplasty; TVP, tricuspid valvuloplasty; CPB, cardiopulmonary bypass; DHCA, hypothermic circulatory arrest; ICU, intensive care unit.

Table 6 Comparison between patients who survived with who did not in the follow-up period

\begin{tabular}{|c|c|c|c|}
\hline Characteristics & Follow-up survivals $(n=529)$ & Follow-up death $(n=40)$ & $P$ value \\
\hline Regular treatment of hypertension (\%) & $182(34.4)$ & $5(12.5)$ & 0.004 \\
\hline \multicolumn{4}{|l|}{ Antihypertensive medications } \\
\hline Beta-blocker (\%) & $132(25.0)$ & $5(12.5)$ & 0.076 \\
\hline Diuretic (\%) & $58(11.0)$ & $1(2.5)$ & 0.108 \\
\hline ACEI or ARB (\%) & $74(14.0)$ & $3(7.5)$ & 0.247 \\
\hline Others (\%) & $50(9.5)$ & $7(17.5)$ & 0.105 \\
\hline Uncontrolled hypertension (\%) & $157(29.7)$ & $15(37.5)$ & 0.299 \\
\hline
\end{tabular}

Data presented as n (\%). ACEI, angiotensin-converting enzyme inhibitors; ARB, angiotensin receptor blocker.

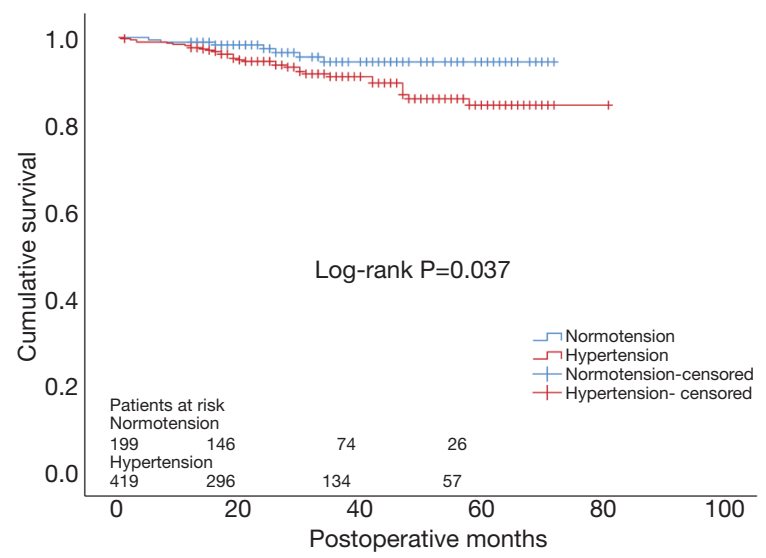

Figure 1 Kaplan-Meier estimate for late survival of patients operated on for type A acute aortic dissection according to the presence of hypertension.
Furthermore, a significant difference of long-term survival rate was discovered by Kaplan-Meier test between hypertensive and normotensive two groups $(\mathrm{P}=0.037)$ (Figure 1).

Following univariate analysis, parameters including age above 65 years $(\mathrm{P}=0.050)$, female gender $(\mathrm{P}=0.095)$, hypertension $(\mathrm{P}=0.044)$, overweight ( $\mathrm{BMI}$ greater than 24) (7) $(\mathrm{P}=0.096)$, duration of hypertension $(\mathrm{P}=0.004)$, diastolic $\mathrm{BP}(\mathrm{P}=0.020)$ and mean $\mathrm{BP}(\mathrm{P}=0.045)$ at admission, postoperative regular treatment of hypertension $(\mathrm{P}=0.015)$, postoperative usage of beta-blockers $(\mathrm{P}=0.073)$ were selected for further multivariate analysis. Multivariable Cox regression analysis showed that hypertension [hazard ratio (HR), 3.040; 95\% confidence interval (CI), 1.124-8.227; $\mathrm{P}=0.029]$, postoperative regular treatment of hypertension (HR, 0.227; 95\% CI, 0.087-0.589; $\mathrm{P}=0.002$ ) and usage of beta-blockers after discharge (HR, 0.328; 95\% CI, 0.126- 
$0.853 ; \mathrm{P}=0.022$ ) were proved to be independent predictors for long-term survival (Table 7).

\section{Discussion}

It has been known that hypertension is one of the most important risk factors for aortic dissection (4). However, the predictive value of hypertension in patients of TA-AAD underwent cardiac surgeries remained understudied. Therefore, we examined the association between preexisting hypertension and mortality rates in our hospital. In this study, we demonstrated that complicating with hypertension did not increase patients' mortality rate within 30 days after surgery. However, the long-term survival rate of hypertensive patients was significantly lower compared to normotensive patients. In addition, hypertension was identified as an independent predictor for increased long-term mortality rate.

Compared to previous studies that trying to decipher the influence of blood pressure on patients with TA-AAD $(8,9)$, one of the strengths of this study was that we applied PSM methods on the cohort before further analysis. As a result, confounders that might bias the results were excluded between two groups. The patient cohort analyzed in our study comprised of consecutively enrolled patients who underwent surgical repair of TA-AAD. In our cohort, 492 patients $(69.1 \%)$ were complicated with hypertension, a percentage that in consistent with previous studies $(5,10-13)$. Our clinical profile depictions suggested that hypertensive patients were older and heavier than normotensive patients, similar to previous reports $(8,9,14)$. Unsurprisingly, hypertensive patients were associated with significantly elevated preoperative sCr, which was also in consistent with other reports $(9,15)$. Elevated preoperative leucocyte levels were also identified in hypertensive patients, which was consistent with prior studies that demonstrated an association between leucocyte count and hypertension incidence $(16,17)$. This finding suggested that inflammation may play a role in the development of hypertension. Most studies started their survival analysis on hospital day 1 thereby included in-hospital deaths. It is important to notice that we only count deaths that happened after hospital discharge in this study which might gave us an underestimated mortality rate when comparing to other studies.

Another important finding in our study was that hypertensive patients received less aortic valve management. However, normotensive patients tend to be younger in our study and we could not rule out the influence of age on disease manifestations and procedure selections. As a matter of fact, reports from IRAD investigators suggested that younger patients more often underwent root surgeries (18).

In the present study, we found that hypertension was not associated with increasing peri-operative mortality rate. Several reports analyzing the influence of preoperative factors on the survival of patients with TA-AAD found a similar result that hypertension was not an independent predictor of higher operative mortality $(8,19-21)$. One reason might be that the patients' $\mathrm{BP}$ was closely monitored and proper anti-hypertensive medications were prescribed during hospitalization period. Another reason might be that the area of greatest aortic shear stress was surgically repaired and the risk of developing a new separation of the aortic vessel layers was minimized.

Our findings suggested hypertension was associated with long-term survival in TA-AAD patients who received surgeries. We also found that patients who had never been treated or taking antihypertensive medication irregularly after surgery had an increased long-term mortality compared to whose hypertension was properly controlled. Uncontrolled BP could lead to cardiac failure and aortic rupture, which were the main reasons for late death as well as other studies (21). Unfortunately, it is difficult to achieve in some patients. From a nationwide database of 276,197 subjects (aged 40-75 years), there were 123,063 hypertensive patients, and only 77,379 (63\%) received proper antihypertensive drugs. Another research found that only 598 out of 848 ( $70.5 \%)$ hypertensive patients received regular medication in China (9). A previous report demonstrated that an increase in $\mathrm{BP}$ of $26 \mathrm{~mm} \mathrm{Hg}$ was equivalent to an increase of the aortic diameter of 1 centimeter (22). An 25 years follow-up study of 252 patients who underwent repair of TA-AAD suggested that the reoperation probability was markedly decreased with improved systolic BP control (11). Tsai and associates examined 303 consecutive patients with TA-AAD concluded that patents who died within 3 years of surgery had increased systolic BP compared with those who survived (130 vs. 122, $\mathrm{P}<0.01$ ) (13). Consistent with previous studies, our data suggested that strict medication adherence and $\mathrm{BP}$ control after discharge is pivotal in the management of patients experienced aortic dissection surgery repair.

Contrary to our conclusions, Merkle and colleagues (8) demonstrated that hypertensive patients were not associated with worse long-term survival compared to normotensive patients. However, compared to their study, we involved more cases and more sophisticated statistical analysis like PSM and Cox regression analysis to eliminate potential bias 
Table 7 Univariate and multivariate analysis on factors for late mortality in hospital survivors

\begin{tabular}{|c|c|c|c|c|c|c|}
\hline Characteristics & \multicolumn{3}{|c|}{ Univariate analysis } & \multicolumn{3}{|c|}{ Multivariate analysis } \\
\hline $\operatorname{Age}(\geq 65 y)$ & 2.051 & $1.001-4.203$ & 0.050 & & $\mathrm{~N} / \mathrm{A}$ & 0.460 \\
\hline Female gender & 1.727 & $0.910-3.275$ & 0.095 & & $\mathrm{~N} / \mathrm{A}$ & 0.084 \\
\hline Hypertension & 2.317 & $1.025-5.238$ & 0.044 & 3.040 & $1.124-8.227$ & 0.029 \\
\hline Duration of hypertension & 1.057 & $1.018-1.098$ & 0.004 & 1.042 & $0.994-1.092$ & 0.087 \\
\hline Regular treatment of hypertension & 0.314 & $0.123-0.801$ & 0.015 & 0.227 & $0.087-0.589$ & 0.002 \\
\hline DBP at admission & 1.028 & $1.004-1.052$ & 0.020 & & $\mathrm{~N} / \mathrm{A}$ & 0.278 \\
\hline Mean BP at admission & 1.021 & $1 . .000-1.042$ & 0.045 & & $\mathrm{~N} / \mathrm{A}$ & 0.428 \\
\hline SBP at admission & 1.010 & $0.996-1.023$ & 0.160 & & $\mathrm{~N} / \mathrm{A}$ & \\
\hline Uncontrolled hypertension & 1.047 & $0.741-2.670$ & 0.296 & & $\mathrm{~N} / \mathrm{A}$ & \\
\hline Calcium channel blocker & 0.503 & $0.179-1.413$ & 0.192 & & $\mathrm{~N} / \mathrm{A}$ & \\
\hline ACEI or ARB & 0.573 & $0.177-1.859$ & 0.354 & & $\mathrm{~N} / \mathrm{A}$ & \\
\hline Other medication & 1.786 & $0.790-4.037$ & 0.164 & & $\mathrm{~N} / \mathrm{A}$ & \\
\hline Previous coronary artery disease & 1.514 & $0.365-6.238$ & 0.567 & & $N / A$ & \\
\hline Cerebrovascular disease & 2.537 & $0.782-8.231$ & 0.121 & & $\mathrm{~N} / \mathrm{A}$ & \\
\hline $\mathrm{sCr}$ & 1.001 & $0.998-1.003$ & 0.559 & & $N / A$ & \\
\hline TAR & 0.719 & $0.351-1.472$ & 0.367 & & $\mathrm{~N} / \mathrm{A}$ & \\
\hline CPB time & 1.000 & $0.995-1.004$ & 0.923 & & $\mathrm{~N} / \mathrm{A}$ & \\
\hline Aortic valve & 1.009 & $0.520-1.955$ & 0.980 & & $\mathrm{~N} / \mathrm{A}$ & \\
\hline DHCA time & 0.980 & $0.957-1.004$ & 0.105 & & $\mathrm{~N} / \mathrm{A}$ & \\
\hline $\begin{array}{l}\text { Drainage volume } 24 \text { hours after } \\
\text { surgery }\end{array}$ & 1.000 & $0.999-1.001$ & 0.901 & & $\mathrm{~N} / \mathrm{A}$ & \\
\hline Ventilation time & 1.000 & $0.992-1.007$ & 0.971 & & $\mathrm{~N} / \mathrm{A}$ & \\
\hline ICU stay time & 1.003 & $0.986-1.019$ & 0.761 & & $\mathrm{~N} / \mathrm{A}$ & \\
\hline Hospital stay time & 1.000 & $0.973-1.028$ & 0.994 & & $\mathrm{~N} / \mathrm{A}$ & \\
\hline
\end{tabular}

HR, hazard ratio; CI, confidence interval; BMI, body mass index; DBP, diastolic blood pressure; SBP, systolic blood pressure; ACEI, angiotensin-converting enzyme inhibitors; ARB, angiotensin receptor blocker; TAR, total arch replacement; SCr, serum creatinine; WBC, white blood cell; PLT, platelet; LVEF, left ventricular ejection fraction; CPB, cardiopulmonary bypass; DHCA, hypothermic circulatory arrest; ICU, intensive care unit; N/A, not available. 
in baseline characteristics, which might render us a better strength. And it is important to point out that ethnics might also play an important role. In addition, the observation in a cohort consisted of 232 consecutive patients treated surgically for TA-AAD followed up to 4 years supported our findings (21). Numerous studies, including us, proved that in order to prevent late adverse events, frequent follow-up and appropriate medication are essential for patients who have undergone aortic repair for TA-AAD.

Regular beta-blockers treatment after hospital discharge was associated with decreased long-term mortality in our study. Current evidence regarding the beneficial effect of beta-blockers may be numerous, while there is little data after aortic dissection repair. In a prospective cohort of 3,102 patients, Chan et al. reported that patients regularly taking beta-blockers after cardiac surgery exhibit a substantially lower mortality rate during long-term follow-up (23). Thus, in patients with hypertension, strong consideration should be given to the prescription of beta-blockers after aortic dissection repair surgery.

\section{Study limitations}

Our research presented with several limitations. Firstly, our study was a retrospective study conducted in a single center, with a cohort that might not necessarily representable for the general population. In addition, our study had a median follow-up period of 29 months. A longer follow-up period might provide additional insights into the impact of hypertension on disease's long-term outcome. Another points that worth mentioning are that the survival analysis excluded patients lost to follow-up and PSM method was not applied to the long-term follow-up analysis. Moreover, during the follow-up, the exact cause of death in some patients could not be verified, which also might affect our analysis.

\section{Conclusions}

Hypertensive patients who underwent surgeries for TAAAD presented with similar perioperative outcomes but increased long-term mortality rate compared to normotensive patients. Hypertension was identified as an independent risk factor for late mortality. Our study provided insights in re-evaluating the importance of hypertension in TA-AAD patients. Future studies included more patients with prolonged follow-up period are needed to verify our findings.

\section{Acknowledgments}

Funding: None.

\section{Footnote}

Reporting Checklist: The authors have completed the STROBE reporting checklist. Available at http://dx.doi. org/10.21037/jtd-20-2336

Data Sharing Statement: Available at http://dx.doi. org/10.21037/jtd-20-2336

Conflicts of Interest: All authors have completed the ICMJE uniform disclosure form (available at http://dx.doi. org/10.21037/jtd-20-2336). The authors have no conflicts of interest to declare.

Ethical Statement: The authors are accountable for all aspects of the work in ensuring that questions related to the accuracy or integrity of any part of the work are appropriately investigated and resolved. The ethics committee of Nanjing Drum Tower hospital approved this retrospective study (No. BL2014004) and waived the need for individual informed consent due to the nature of this study. The study was conducted in accordance with the Declaration of Helsinki (as revised in 2013).

Open Access Statement: This is an Open Access article distributed in accordance with the Creative Commons Attribution-NonCommercial-NoDerivs 4.0 International License (CC BY-NC-ND 4.0), which permits the noncommercial replication and distribution of the article with the strict proviso that no changes or edits are made and the original work is properly cited (including links to both the formal publication through the relevant DOI and the license). See: https://creativecommons.org/licenses/by-nc-nd/4.0/.

\section{References}

1. Sheikh AS, Ali K, Mazhar S. Acute aortic syndrome. Circulation 2013;128:1122-7.

2. Trimarchi S, Nienaber CA, Rampoldi V. Contemporary Results of Surgery in Acute Type A Aortic Dissection: The International Registry of Acute Aortic Dissection Experience. J Thorac Cardiovasc Surg 2005;129:112-22.

3. Erbel R, Aboyans V, Boileau C, et al. 2014 ESC Guidelines 
on the diagnosis and treatment of aortic diseases:

Document covering acute and chronic aortic diseases of the thoracic and abdominal aorta of the adult. The Task Force for the Diagnosis and Treatment of Aortic Diseases of the European Society of Cardiology (ESC). Eur Heart J 2014;35:2873-926.

4. Landenhed M, Engstrom G, Gottsater A, et al. Risk profiles for aortic dissection and ruptured or surgically treated aneurysms: a prospective cohort study. J Am Heart Assoc 2015;4:e001513.

5. Chan KK, Rabkin SW. Increasing prevalence of hypertension among patients with thoracic aorta dissection: trends over eight decades--a structured metaanalysis. Am J Hypertens 2014;27:907-17.

6. Rosendorff C, Lackland DT, Allison M, et al. Treatment of hypertension in patients with coronary artery disease: A scientific statement from the American Heart Association, American College of Cardiology, and American Society of Hypertension. J Am Soc Hypertens 2015;9:453-98.

7. Chen C, Lu FC. The guidelines for prevention and control of overweight and obesity in Chinese adults. Biomed Environ Sci 2004;17 Suppl:1-36.

8. Merkle J, Sabashnikov A, Deppe AC, et al. Impact of hypertension on early outcomes and long-term survival of patients undergoing aortic repair with Stanford A dissection. Perfusion 2018;33:463-71.

9. Otaki Y, Watanabe T, Konta T, et al. Effect of Hypertension on Aortic Artery Disease-Related Mortality3.8-Year Nationwide Community-Based Prospective Cohort Study. Circ J 2018;82:2776-82.

10. Booher AM, Isselbacher EM, Nienaber CA, et al. The IRAD classification system for characterizing survival after aortic dissection. Am J Med 2013;126:730.e19-24.

11. Melby SJ, Zierer A, Damiano RJ, Jr., et al. Importance of blood pressure control after repair of acute type a aortic dissection: 25 -year follow-up in 252 patients. J Clin Hypertens (Greenwich) 2013;15:63-8.

12. Dong N, Piao H, Li B, et al. Poor management of hypertension is an important precipitating factor for the development of acute aortic dissection. J Clin Hypertens (Greenwich) 2019;21:804-12.

13. Tsai TT, Evangelista A, Nienaber CA, et al. Longterm survival in patients presenting with type A acute aortic dissection: insights from the International Registry of Acute Aortic Dissection (IRAD). Circulation 2006;114:1350-6.
14. Trimarchi S, Eagle KA, Nienaber CA, et al. Role of age in acute type A aortic dissection outcome: report from the International Registry of Acute Aortic Dissection (IRAD). J Thorac Cardiovasc Surg 2010;140:784-9.

15. Eguchi K, Miyashita H, Takenaka T, et al. High central blood pressure is associated with incident cardiovascular events in treated hypertensives: the ABC-J II Study. Hypertens Res 2018;41:947-56.

16. Shankar A, Klein BE, Klein R. Relationship between white blood cell count and incident hypertension. Am J Hypertens 2004;17:233-9.

17. Tatsukawa Y, Hsu WL, Yamada M, et al. White blood cell count, especially neutrophil count, as a predictor of hypertension in a Japanese population. Hypertens Res 2008;31:1391-7.

18. Januzzi JL, Isselbacher EM, Fattori R, et al. Characterizing the young patient with aortic dissection: results from the International Registry of Aortic Dissection (IRAD). J Am Coll Cardiol 2004;43:665-9.

19. Ehrlich M, Fang WC, Grabenwoger M, et al. Perioperative risk factors for mortality in patients with acute type A aortic dissection. Circulation 1998;98:II294-8.

20. Apaydin AZ, Buket S, Posacioglu H, et al. Perioperative risk factors for mortality in patients with acute type A aortic dissection. Ann Thorac Surg 2002;74:2034-9; discussion 2039.

21. Bekkers JA, Raap GB, Takkenberg JJ, et al. Acute type A aortic dissection: long-term results and reoperations. Eur J Cardiothorac Surg 2013;43:389-96.

22. Okamoto RJ, Xu H, Kouchoukos NT, et al. The influence of mechanical properties on wall stress and distensibility of the dilated ascending aorta. J Thorac Cardiovasc Surg 2003;126:842-50.

23. Chan AY, McAlister FA, Norris CM, et al. Effect of betablocker use on outcomes after discharge in patients who underwent cardiac surgery. J Thorac Cardiovasc Surg 2010;140:182-7, 187.e1.

Cite this article as: Wang Z, Ge M, Chen T, Chen C, Zong Q, Lu L, Wang D. Impact of hypertension on short- and longterm survival of patients who underwent emergency surgery for type A acute aortic dissection. J Thorac Dis 2020;12(11):66186628. doi: $10.21037 /$ jtd-20-2336 